QUARTERLY OF APPLIED MATHEMATICS

VOLUME LXIII, NUMBER 3

SEPTEMBER 2005, PAGES 553-573

S $0033-569 X(05) 00974-0$

Article electronically published on July 11, 2005

\title{
LOCAL UNIQUENESS AND CONTINUATION OF SOLUTIONS FOR THE DISCRETE COULOMB FRICTION PROBLEM IN ELASTOSTATICS
}

\author{
BY \\ PATRICK HILD (Laboratoire de Mathématiques de Besançon, CNRS UMR 6623, Université de \\ Franche-Comté, 16 route de Gray, 25030 Besançon Cedex, France) \\ AND \\ YVES RENARD (MIP, CNRS UMR 5640, INSAT, Complexe scientifique de Rangueil, 31077 \\ Toulouse, France)
}

We dedicate this article to the memory of Jean-Claude Paumier.

Abstract. This work is concerned with the frictional contact problem governed by the Signorini contact model and the Coulomb friction law in static linear elasticity. We consider a general finite-dimensional setting and we study local uniqueness and smooth or nonsmooth continuation of solutions by using a generalized version of the implicit function theorem involving Clarke's gradient. We show that for any contact status there exists an eigenvalue problem and that the solutions are locally unique if the friction coefficient is not an eigenvalue. Finally we illustrate our general results with a simple example in which the bifurcation diagrams are exhibited and discussed.

Introduction. Friction problems are of current interest both from the theoretical and practical point of view in structural mechanics. Numerous studies deal with the widespread Coulomb friction law [6] introduced in the eighteenth century which takes into account the possibility of slip and stick on the friction area. Generally the friction model is coupled with a contact law, and very often one considers the unilateral contact allowing separation and contact and excluding interpenetration. Although quite simple in its formulation, the Coulomb friction law shows great mathematical difficulties which have not allowed a complete understanding of the model. In the simple case of continuum elastostatics (i.e., the so-called static friction law) only existence results for small friction

Received January 13, 2005.

2000 Mathematics Subject Classification. Primary 74M10, 74G20; Secondary 35A07, 35J85, 65N30.

Key words and phrases. Coulomb friction, unilateral contact, local uniqueness, bifurcation, Clarke's gradient.

E-mail address: hild@math.univ-fcomte.fr

E-mail address: renard@insa-toulouse.fr

(C)2005 Brown University 
coefficients have been obtained (see [24, 19, 9]) as well as some examples of nonuniqueness of solutions for large friction coefficients [14, 15. As far as we know there does not exist any uniqueness result and/or nonexistence example for the continuous model.

In the finite-dimensional context, the finite element problem admits always a solution which is unique provided that the friction coefficient is lower than a critical value vanishing when the discretization parameter $h$ tends to zero (see e.g., 10]). In fact the critical value behaves like $h^{1 / 2}$. Actually, it is not established if this mesh-size-dependent bound ensuring uniqueness represents a real loss of uniqueness or if it comes from a "nonoptimal" mathematical analysis. In particular we don't know if for a given geometry there may exist a mesh and a nonuniqueness example for an arbitrary small friction coefficient. Several examples of nonunique solutions exist for the static case involving a finite or infinite number of solutions (see, e.g., [13]). Moreover it is possible to find (using finite element computations) for an arbitrary small friction coefficient a geometry with a nonuniqueness example (see [12]).

Our aim in this paper is to propose and to study a framework for the finite-dimensional problem in order to obtain results ensuring local uniqueness and smooth or nonsmooth continuation of solutions. As far as we know the only existing results concerned with uniqueness in the finite-dimensional case are global and assume that the friction coefficient is small. As a consequence there does not exist any uniqueness result for large friction coefficients. Roughly speaking our method can be summarized as follows. We use a formulation of the frictional contact conditions (without any regularization or smoothing) following the ideas introduced in [22] in which the discrete problem is written $\mathcal{H}(\mathcal{F}, Y)=0$ where $\mathcal{F}$ is the friction coefficient and $Y$ is a vector comprising the displacement field as well as the normal and tangential loads on the contact zone. Having at our disposal a solution $(\mathcal{F}, Y)$ to the discrete problem we obtain an eigenvalue problem depending on the status of the nodes on the contact zone. The eigenvalue problem comes from the application of the generalized implicit function theorem involving Clarke's gradient, which is well-adapted to the unilateral contact model with Coulomb friction. We write the eigenvalue problem both in the smooth case (when $\mathcal{H}$ is $\mathcal{C}^{1}$ near $(\mathcal{F}, Y)$ ) and in the nonsmooth case (when $\mathcal{H}$ is only Lipschitz-continuous near $(\mathcal{F}, Y)$ ). The main result obtained in this paper is that the solution $(\mathcal{F}, Y)$ is locally unique if the friction coefficient is not an eigenvalue.

The paper is outlined as follows. Section 1 deals with the setting of the frictional contact problem in linear elasticity, and the weak formulations are derived in Section 2. Section 3 contains the main results: a first one dealing with local uniqueness and $\mathcal{C}^{1}$ continuation of solutions in the regular case and a second one concerning local uniqueness and Lipschitz continuation of solutions in the nonregular case (i.e., when some points on the contact zone satisfy grazing contact or vanishing slip conditions). We prove that for any contact status there exists an eigenvalue problem and that the solutions are locally unique if the friction coefficient is not an eigenvalue. Section 4 is concerned with some explicit calculus in the case of a single finite element mesh. The different types of bifurcation points (causing the loss of local uniqueness) where the eigenvalues are located are exhibited and discussed. 


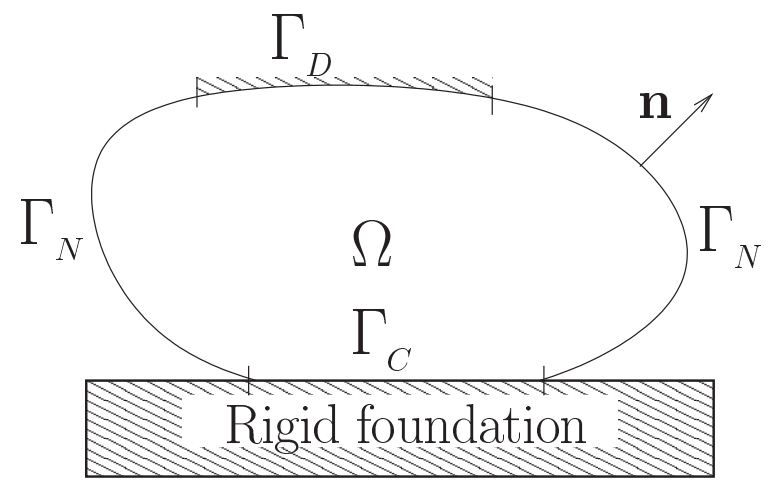

Fig. 1. Elastic body $\Omega$ in frictional contact

1. Problem set up. Let $\Omega \subset \mathbb{R}^{d}(d=2$ or 3 ) be a bounded domain representing the reference configuration of a linearly elastic body whose boundary $\partial \Omega$ consists of three nonoverlapping open parts $\Gamma_{N}, \Gamma_{D}$ and $\Gamma_{C}$ with $\overline{\Gamma_{N}} \cup \overline{\Gamma_{D}} \cup \overline{\Gamma_{C}}=\partial \Omega$. We assume that the measures of $\Gamma_{C}$ and $\Gamma_{D}$ are positive. The body is submitted to a Neumann condition on $\Gamma_{N}$, a Dirichlet condition on $\Gamma_{D}$ and a unilateral contact condition with static Coulomb friction between the body and a flat rigid foundation on $\Gamma_{C}$ (see Fig. 1).

The frictional contact problem consists in finding the displacement field $u=u(x), x \in$ $\Omega$, satisfying:

$$
\begin{array}{rc}
-\operatorname{div} \sigma(u)=f, & \text { in } \Omega, \\
\sigma(u)=\mathcal{A} \varepsilon(u), & \text { in } \Omega, \\
\sigma(u) \mathbf{n}=g, & \text { on } \Gamma_{N}, \\
u=0, & \text { on } \Gamma_{D},
\end{array}
$$

where $\sigma(u)$ denotes the stress tensor, $\varepsilon(u)$ stands for the linearized strain tensor, $\mathcal{A}$ is the elastic coefficient tensor which satisfies the classical conditions of symmetry and ellipticity. The notation $\mathbf{n}$ represents the unit outward normal to $\Omega$ on $\partial \Omega$, and $g, f$ are the given external loads.

On $\partial \Omega$, it is usual to decompose the displacement and the stress vectors into normal and tangential components as follows:

$$
\begin{gathered}
u_{N}=u \cdot \mathbf{n}, \quad u_{T}=u-u_{N} \mathbf{n}, \\
\sigma_{N}(u)=(\sigma(u) \mathbf{n}) . \mathbf{n}, \quad \sigma_{T}(u)=\sigma(u) \mathbf{n}-\sigma_{N}(u) \mathbf{n} .
\end{gathered}
$$

To give a clear sense to this decomposition, we assume that $\Gamma_{C}$ is $\mathcal{C}^{1}$ regular. Supposing also that there is no initial gap between the solid and the rigid foundation, the unilateral contact condition is expressed by the following complementary condition on $\Gamma_{C}$ :

$$
u_{N} \leq 0, \sigma_{N}(u) \leq 0, u_{N} \sigma_{N}(u)=0 .
$$


Denoting by $\mathcal{F}$ the nonnegative friction coefficient, the static Coulomb friction condition on $\Gamma_{C}$ reads as:

$$
\begin{array}{ll}
\text { if } u_{T}=0, \quad \text { then } \quad & \left|\sigma_{T}(u)\right| \leq-\mathcal{F} \sigma_{N}(u), \\
\text { if } u_{T} \neq 0, \quad \text { then } \quad \sigma_{T}(u)=\mathcal{F} \sigma_{N}(u) \frac{u_{T}}{\left|u_{T}\right|} .
\end{array}
$$

When the friction coefficient vanishes on $\Gamma_{C}$, then the friction conditions merely become $\sigma_{T}(u)=0$ on $\Gamma_{C}$.

\section{Weak formulations.}

2.1. Classical weak formulation. We present here the classical weak formulation proposed by G. Duvaut [7]. Let us introduce the following Hilbert spaces:

$$
\begin{gathered}
V=\left\{v \in H^{1}\left(\Omega ; \mathbb{R}^{d}\right), v=0 \text { on } \Gamma_{D}\right\}, \\
X=\left\{v_{\Gamma_{C}}: v \in V\right\} \subset H^{1 / 2}\left(\Gamma_{C} ; \mathbb{R}^{d}\right), \\
X_{N}=\left\{\left.v_{N}\right|_{\Gamma_{C}}: v \in V\right\}, \quad X_{T}=\left\{\left.v_{T}\right|_{\Gamma_{C}}: v \in V\right\},
\end{gathered}
$$

and their topological dual spaces $V^{\prime}, X^{\prime}, X_{N}^{\prime}$ and $X_{T}^{\prime}$. It is assumed that $\Gamma_{C}$ is sufficiently smooth such that $X_{N} \subset H^{1 / 2}\left(\Gamma_{C}\right), X_{T} \subset H^{1 / 2}\left(\Gamma_{C} ; \mathbb{R}^{d-1}\right), X_{N}^{\prime} \subset H^{-1 / 2}\left(\Gamma_{C}\right)$ and $X_{T}^{\prime} \subset H^{-1 / 2}\left(\Gamma_{C} ; \mathbb{R}^{d-1}\right)$.

Classically, $H^{1 / 2}\left(\Gamma_{C}\right)$ is the space of the restrictions on $\Gamma_{C}$ of traces on $\partial \Omega$ of functions of $H^{1}(\Omega)$, and $H^{-1 / 2}\left(\Gamma_{C}\right)$ is the dual space of $H_{00}^{1 / 2}\left(\Gamma_{C}\right)$, which is the space of the restrictions on $\Gamma_{C}$ of functions of $H^{1 / 2}(\partial \Omega)$ vanishing outside $\Gamma_{C}$. We refer to [23, 1 for a detailed study concerning trace operators and to [21, 11] for a presentation of the trace operators involved in contact problems.

Now, the set of admissible displacements is defined as

$$
K=\left\{v \in V, v_{N} \leq 0 \text { on } \Gamma_{C}\right\} .
$$

The following functionals

$$
\begin{gathered}
a(u, v)=\int_{\Omega} \mathcal{A} \varepsilon(u): \varepsilon(v) d x, \\
l(v)=\int_{\Omega} f \cdot v d x+\int_{\Gamma_{N}} g \cdot v d \Gamma, \\
j\left(\lambda_{N}, v_{T}\right)=-\left\langle\mathcal{F} \lambda_{N},\left|v_{T}\right|\right\rangle_{X_{N}^{\prime}, X_{N}}
\end{gathered}
$$

represent respectively the virtual work of elastic forces, the external loads and the "virtual work" of friction forces. We assume the following standard hypotheses:

$a(.,$.$) is a bilinear symmetric V$-elliptic and continuous form on $V \times V$ :

$$
\exists \alpha>0, \exists M>0, a(v, v) \geq \alpha\|v\|_{V}^{2}, a(u, v) \leq M\|u\|_{V}\|v\|_{V} \forall u, v \in V,
$$

$l($.$) is a linear continuous form on V$,

$\mathcal{F}$ is a Lipschitz-continuous nonnegative function on $\Gamma_{C}$. 
Condition (2.3) ensures that $j\left(\lambda_{N}, v_{T}\right)$ is linear continuous with respect to $\lambda_{N}$ and convex and lower semi-continuous with respect to $v_{T}$ when $\lambda_{N}$ is a nonpositive element of $X_{N}^{\prime}$ (see for instance [3). Problem (1.1)-(1.7) is then formally equivalent to the following inequality formulation $([7,8])$ :

$$
\left\{\begin{array}{l}
\text { Find } u \in K \text { satisfying } \\
a(u, v-u)+j\left(\sigma_{N}(u), v_{T}\right)-j\left(\sigma_{N}(u), u_{T}\right) \geq l(v-u), \quad \forall v \in K .
\end{array}\right.
$$

The first existence results for this problem were obtained by Nečas, Jarušek and Haslinger in 24 for a two-dimensional elastic strip, assuming that the coefficient of friction is small enough and using a shifting technique, previously introduced by Fichera, and later applied to more general domains by Jarušek [19. Eck and Jarušek [9] give a different proof using a penalization method. We emphasize that most results on existence for frictional problems involve a condition of smallness for the friction coefficient (and a compact support of the friction coefficient on $\Gamma_{C}$ ). It has been proven recently that the problem (1.1)-(1.7) does not admit a unique solution in the general case. More precisely there exist for some loads an infinity of solutions slipping in the same direction for precise friction coefficients (see [15) and there exist for some loads at least two isolated solutions involving stick and separation for large friction coefficients (see [14]).

The main difficulty in (2.4) comes from the coupling between the friction threshold and the contact pressure $\sigma_{N}(u)$. The consequence is that this problem does not represent a variational inequality, in the sense that it cannot be derived from an optimization problem.

2.2. Hybrid weak formulation. Let $K_{N}$ be the set of admissible normal displacements on $\Gamma_{C}$ :

$$
K_{N}=\left\{v_{N} \in X_{N}: v_{N} \leq 0 \text { a.e. on } \Gamma_{C}\right\} .
$$

The set of admissible normal stresses on $\Gamma_{C}$ is defined by

$$
\Lambda_{N}=\left\{f_{N} \in X_{N}^{\prime}:\left\langle f_{N}, v_{N}\right\rangle_{X_{N}^{\prime}, X_{N}} \geq 0, \quad \forall v_{N} \in K_{N}\right\} .
$$

This is the opposite of $K_{N}^{*}$, the polar cone to $K_{N}$. Given $\lambda_{N} \in \Lambda_{N}$ the set of admissible tangential stresses on $\Gamma_{C}$ can be defined as

$$
\Lambda_{T}\left(\mathcal{F} \lambda_{N}\right)=\left\{f_{T} \in X_{T}^{\prime}:-\left\langle f_{T}, w_{T}\right\rangle_{X_{T}^{\prime}, X_{T}}+\left\langle\mathcal{F} \lambda_{N},\left|w_{T}\right|\right\rangle_{X_{N}^{\prime}, X_{N}} \leq 0, \forall w_{T} \in X_{T}\right\} .
$$

With this notation, the formulation (2.4) is equivalent to the following problem:

$$
\left\{\begin{array}{l}
\text { find } u \in V, \lambda_{N} \in X_{N}^{\prime} \text { and } \lambda_{T} \in X_{T}^{\prime} \text { satisfying } \\
a(u, v)=l(v)+\left\langle\lambda_{N}, v_{N}\right\rangle_{X_{N}^{\prime}, X_{N}}+\left\langle\lambda_{T}, v_{T}\right\rangle_{X_{T}^{\prime}, X_{T}}, \forall v \in V, \\
\lambda_{N} \in \Lambda_{N},\left\langle\mu_{N}-\lambda_{N}, u_{N}\right\rangle_{X_{N}^{\prime}, X_{N}} \geq 0, \forall \mu_{N} \in \Lambda_{N}, \\
\lambda_{T} \in \Lambda_{T}\left(\mathcal{F} \lambda_{N}\right),\left\langle\mu_{T}-\lambda_{T}, u_{T}\right\rangle_{X_{T}^{\prime}, x_{T}} \geq 0, \forall \mu_{T} \in \Lambda_{T}\left(\mathcal{F} \lambda_{N}\right) .
\end{array}\right.
$$

It is easy to see that $\lambda_{N}=\sigma_{N}(u)$ and $\lambda_{T}=\sigma_{T}(u)$ at least in a weak sense. 
2.3. Projection formulation. A solution $u, \lambda_{N}, \lambda_{T}$ to Problem (2.5) which satisfies $\lambda_{N} \in L^{2}\left(\Gamma_{C}\right)$ (and then $\lambda_{T} \in L^{2}\left(\Gamma_{C} ; \mathbb{R}^{d-1}\right)$ ) is also a solution to the following problem (see [22]) for an arbitrary $r>0$ :

$$
\left\{\begin{array}{l}
\text { find } u \in V, \lambda_{N} \in X_{N}^{\prime} \text { and } \lambda_{T} \in X_{T}^{\prime} \text { satisfying } \\
a(u, v)=l(v)+\left\langle\lambda_{N}, v_{N}\right\rangle_{X_{N}^{\prime}, X_{N}}+\left\langle\lambda_{T}, v_{T}\right\rangle_{X_{T}^{\prime}, X_{T}}, \forall v \in V \\
\lambda_{N}=P_{\Lambda_{N}}\left(\lambda_{N}-r u_{N}\right), \\
\lambda_{T}=P_{\Lambda_{T}\left(\mathcal{F} \lambda_{N}\right)}\left(\lambda_{T}-r u_{T}\right),
\end{array}\right.
$$

where $P_{\Lambda_{N}}$ and $P_{\Lambda_{T}\left(\mathcal{F} \lambda_{N}\right)}$ stand for the $L^{2}\left(\Gamma_{C}\right)$-projection operators onto $\Lambda_{N}$ and $\Lambda_{T}\left(\mathcal{F} \lambda_{N}\right)$, respectively.

REMARK 2.1. The previous formulation does not involve any regularization of the frictional contact conditions. For a given $r>0$ it is equivalent to Problem (2.5). Therefore the solution $u, \lambda_{N}, \lambda_{T}$ does not depend on $r$.

2.4. Hybrid finite element discretization. Classically, let $V^{h} \subset V$ be a family of finitedimensional sub-vector spaces indexed by $h$ coming from a regular finite element discretization of the domain $\Omega$ (the notation $h$ represents the diameter of the largest element). We define

$$
\begin{gathered}
X_{N}^{h}=\left\{v_{\left.N\right|_{\Gamma_{C}} ^{h}}: v^{h} \in V^{h}\right\}, \\
X_{T}^{h}=\left\{v_{\left.T\right|_{\Gamma_{C}} ^{h}}: v^{h} \in V^{h}\right\}, \\
X^{h}=\left\{\left.v^{h}\right|_{\Gamma_{C}}: v^{h} \in V^{h}\right\}=X_{N}^{h} \times X_{T}^{h} .
\end{gathered}
$$

Let us denote also by $X_{N}^{\prime h} \subset X_{N}^{\prime} \cap L^{2}\left(\Gamma_{C}\right), X_{T}^{\prime h} \subset X_{T}^{\prime} \cap L^{2}\left(\Gamma_{C} ; \mathbb{R}^{d-1}\right)$ and $X^{\prime h}=$ $X_{N}^{\prime h} \times X_{T}^{\prime h}$ some finite element approximations of $X_{N}^{\prime}, X_{T}^{\prime}$ and $X^{\prime}$ respectively satisfying the Babuska-Brezzi "inf-sup" condition:

$$
\inf _{\lambda^{h}=\left(\lambda_{N}^{h}, \lambda_{T}^{h}\right) \in X^{\prime h}} \sup _{v^{h} \in V^{h}} \frac{\int_{\Gamma_{C}} \lambda_{N}^{h} v_{N}^{h} d \Gamma+\int_{\Gamma_{C}} \lambda_{T}^{h} \cdot v_{T}^{h} d \Gamma}{\left\|\lambda^{h}\right\|_{X^{\prime} h}\left\|v^{h}\right\|_{V}} \geq c>0,
$$

where $c$ may depend on $h$.

REMARK 2.2. The inf-sup condition is satisfied if, e.g., $V^{h}$ is a space of continuous finite elements of degree $k \geq 1$ and if $X^{\prime h}$ coincides with the restriction on $\Gamma_{C}$ of functions in $V^{h}$ (see [4]).

Then we choose $\Lambda_{N}^{h} \subset X_{N}^{\prime h}$ and $\Lambda_{T}^{h}\left(\mathcal{F} \lambda_{N}^{h}\right) \subset X_{T}^{\prime h}$ as closed convex approximations of $\Lambda_{N}$ and $\Lambda_{T}\left(\mathcal{F} \lambda_{N}\right)$ respectively (note that the conditions $\Lambda_{N}^{h} \subset \Lambda_{N}$ and $\Lambda_{T}^{h}\left(\mathcal{F} \lambda_{N}^{h}\right) \subset$ $\Lambda_{T}\left(\mathcal{F} \lambda_{N}\right)$ are generally not satisfied). With this notation, the discrete formulation of 
(2.5) becomes

We set

$$
\left\{\begin{array}{l}
\text { find } u^{h} \in V^{h}, \lambda_{N}^{h} \in \Lambda_{N}^{h} \text { and } \lambda_{T}^{h} \in \Lambda_{T}^{h}\left(\mathcal{F} \lambda_{N}^{h}\right) \text { satisfying } \\
a\left(u^{h}, v^{h}\right)=l\left(v^{h}\right)+\int_{\Gamma_{C}} \lambda_{N}^{h} v_{N}^{h} d \Gamma+\int_{\Gamma_{C}} \lambda_{T}^{h} \cdot v_{T}^{h} d \Gamma, \forall v^{h} \in V^{h}, \\
\int_{\Gamma_{C}}\left(\mu_{N}^{h}-\lambda_{N}^{h}\right) u_{N}^{h} d \Gamma \geq 0, \forall \mu_{N}^{h} \in \Lambda_{N}^{h}, \\
\int_{\Gamma_{C}}\left(\mu_{T}^{h}-\lambda_{T}^{h}\right) \cdot u_{T}^{h} d \Gamma \geq 0, \forall \mu_{T}^{h} \in \Lambda_{T}^{h}\left(\mathcal{F} \lambda_{N}^{h}\right) .
\end{array}\right.
$$

$$
u^{h}(x)=\sum_{i=1}^{k_{1}} u^{i} \varphi_{i}(x), \quad \lambda_{N}^{h}(x)=\sum_{i=1}^{k_{2}} \lambda_{N}^{i} \psi_{i}(x), \quad \lambda_{T}^{h}(x)=\sum_{i=1}^{k_{3}} \lambda_{T}^{i} \xi_{i}(x),
$$

where

$$
U=\left(u_{i}\right)_{i=1 . . k_{1}}, \quad L_{N}=\left(\lambda_{N}^{i}\right)_{i=1 . . k_{2}}, \quad L_{T}=\left(\lambda_{T}^{i}\right)_{i=1 . . k_{3}},
$$

and $\varphi_{i}, \psi_{i}$ and $\xi_{i}$ are the shape functions of the chosen finite element method. We introduce the following matrices:

$$
\begin{aligned}
\left(B_{N}\right)_{i j} & =\int_{\Gamma_{C}} \psi_{i} \mathbf{n} \cdot \varphi_{j} d \Gamma, 1 \leq i \leq k_{2}, 1 \leq j \leq k_{1}, \\
\left(B_{T}\right)_{i j} & =\int_{\Gamma_{C}} \xi_{i} \cdot \varphi_{j} d \Gamma, 1 \leq i \leq k_{3}, 1 \leq j \leq k_{1}, \\
B & =\left(\begin{array}{c}
B_{N} \\
B_{T}
\end{array}\right), \\
(K)_{i j} & =a\left(\varphi_{i}, \varphi_{j}\right), \quad 1 \leq i, j \leq k_{1} .
\end{aligned}
$$

The contact condition

$$
\lambda_{N}^{h} \in \Lambda_{N}^{h}, \quad \int_{\Gamma_{C}}\left(\mu_{N}^{h}-\lambda_{N}^{h}\right) u_{N}^{h} d \Gamma \geq 0, \forall \mu_{N}^{h} \in \Lambda_{N}^{h},
$$

can be expressed in a matrix formulation

$$
L_{N} \in \bar{\Lambda}_{N}^{h}, \quad\left(M_{N}-L_{N}\right)^{T} B_{N} U \geq 0, \forall M_{N} \in \bar{\Lambda}_{N}^{h},
$$

where $\bar{\Lambda}_{N}^{h}$ is the convex set of vectors whose components are the nodal values on $\Gamma_{C}$ of functions in $\Lambda_{N}^{h}$ :

$$
\bar{\Lambda}_{N}^{h}=\left\{L_{N}=\left(\lambda_{N}^{i}\right)_{1 \leq i \leq k_{2}} \in \mathbb{R}^{k_{2}}: \sum_{i=1}^{k_{2}} \lambda_{N}^{i} \psi_{i} \in \Lambda_{N}^{h}\right\} .
$$

The expression (2.14) is equivalent to the condition " $-B_{N} U$ belongs to the normal cone to $\bar{\Lambda}_{N}^{h}$ at $L_{N}$ " or equivalently

$$
L_{N}=P_{\bar{\Lambda}_{N}^{h}}\left(L_{N}-r B_{N} U\right),
$$


for any $r>0$. The notation $P_{\bar{\Lambda}_{N}^{h}}$ stands for the projection operator onto $\bar{\Lambda}_{N}^{h}$ with respect to the Euclidean inner product in $\mathbb{R}^{k_{2}}$.

If $L_{N} \in \bar{\Lambda}_{N}^{h}$ is the vector containing the nodal values of $\lambda_{N}^{h} \in \Lambda_{N}^{h}$ we define:

$$
\bar{\Lambda}_{T}^{h}\left(\mathcal{F} L_{N}\right)=\left\{L_{T}=\left(\lambda_{T}^{i}\right)_{1 \leq i \leq k_{3}} \in \mathbb{R}^{k_{3}}: \sum_{i=1}^{k_{3}} \lambda_{T}^{i} \xi_{i} \in \Lambda_{T}^{h}\left(\mathcal{F} \lambda_{N}^{h}\right)\right\}
$$

and the friction condition incorporated into (2.7) can be written (with obvious notation):

$$
L_{T}=P_{\bar{\Lambda}_{T}^{h}\left(\mathcal{F} L_{N}\right)}\left(L_{T}-r B_{T} U\right) .
$$

Therefore the matrix formulation of Problem (2.7) becomes:

$$
\left\{\begin{array}{l}
\text { for a given } r>0, \text { find } U \in \mathbb{R}^{k_{1}}, L_{N} \in \mathbb{R}^{k_{2}} \text { and } L_{T} \in \mathbb{R}^{k_{3}} \text { satisfying } \\
K U=F+B_{N}^{T} L_{N}+B_{T}^{T} L_{T}, \\
L_{N}=P_{\bar{\Lambda}_{N}^{h}}\left(L_{N}-r B_{N} U\right) \\
L_{T}=P_{\bar{\Lambda}_{T}^{h}\left(\mathcal{F} L_{N}\right)}\left(L_{T}-r B_{T} U\right) .
\end{array}\right.
$$

The advantage of this formulation is that contact and friction conditions are expressed without constraints and with Lipschitz-continuous expressions.

The existence of solutions to Problem (2.15) is established in 20] for any friction coefficient together with a uniqueness result when the friction coefficient is small. In this reference the denomination "small" depends on the discretization parameter $h$, and the bound ensuring uniqueness vanishes as $h$ tends towards zero. Although no specific regularity for the mapping $G \longmapsto \bar{\Lambda}_{T}^{h}(G)$ is necessary to prove existence and uniqueness except that $\bar{\Lambda}_{T}^{h}(G)$ has to be a closed convex set, we assume that the following additional property holds:

$$
(X, G) \longmapsto P_{\bar{\Lambda}_{T}^{h}(G)}(X) \text { is Lipschitz-continuous and piecewise } \mathcal{C}^{1} .
$$

This is not a restricting hypothesis since all known discretizations of the Coulomb friction condition satisfy (2.16). In particular this ensures the existence of Clarke's generalized derivative.

2.5. Example of discretization with a Lagrange finite element method. Let us denote by $a_{i}, i=1, \ldots, N_{c}$ the set of all the finite element nodes and by $I_{C}=\left\{i: a_{i} \in \Gamma_{C}\right\}$ the indices of nodes on $\Gamma_{C}$. We still use the notations defined in (2.8)-(2.13) and we suppose that the positive friction coefficient $\mathcal{F}$ is constant on $\Gamma_{C}$. For $i \in I_{C}$ we define a vector $N_{i} \in \mathbb{R}^{k_{1}}$ such that the normal displacement at $a_{i}$ can be written

$$
u_{N}^{h}\left(a_{i}\right)=u_{N}^{i}=N_{i}^{T} U
$$

Similarly, for the tangential displacement, we define for any $i \in I_{C}$ a $k_{1}$-by- $(d-1)$ matrix $T_{i}$ and a $d$-by- $(d-1)$ matrix $t_{i}$ such that

$$
u_{T}\left(a_{i}\right)=u_{T}^{i}=t_{i} T_{i}^{T} U,
$$


where the columns of the (small) matrix $t_{i}$ form an orthonormal basis of the tangent plane to $\Gamma_{C}$ at the node $a_{i}$.

We consider here a space $V^{h}$ of continuous finite element functions of degree $k \geq 1$ with $X_{N}^{\prime h}=X_{N}^{h}$ and $X_{T}^{\prime} h=X_{T}^{h}$. This choice always ensures the fulfillment of the inf-sup condition (2.6). Let us define the following convex cone:

$$
\Lambda_{N}^{h}=\left\{\mu_{N}^{h} \in X_{N}^{h}: \int_{\Gamma_{C}} \mu_{N}^{h} \psi_{i} d \Gamma \leq 0,1 \leq i \leq k_{2}\right\} .
$$

Note that this choice on the multipliers (which means that the generalized loads at the contact nodes are nonpositive) implies that the dual unknowns (e.g., the normal displacements on $\Gamma_{C}$ ) satisfy the nonpenetration condition only at the nodes. If $g \in \Lambda_{N}^{h}$, we choose $\Lambda_{T}^{h}(g)$ as follows:

$$
\Lambda_{T}^{h}(g)=\left\{\mu_{T}^{h} \in X_{T}^{h}:-\int_{\Gamma_{C}} \mu_{T}^{h} \cdot w_{T}^{h} d \Gamma+\sum_{j \in I_{C}} \int_{\Gamma_{C}} g\left|w_{T}^{h}\left(a_{j}\right)\right| \psi_{n_{j}} d \Gamma \leq 0, \forall w_{T}^{h} \in X_{T}^{h}\right\},
$$

where it is assumed that $\psi_{n_{j}}\left(a_{i}\right)=\delta_{i j}, \forall i, j \in I_{C}$. This choice means that the generalized loads at the contact nodes belong to Coulomb's cone. We consider the vectors $B_{N}^{T} L_{N}$ (resp. $B_{T}^{T} L_{T}$ ) containing the generalized normal (resp. tangential) forces at the contact nodes and we denote by $\tilde{\lambda}_{N}^{i}$ (resp. $\tilde{\lambda}_{T}^{i}$ ) those values such that $B_{N}^{T} L_{N}=\sum_{i \in I_{C}} \tilde{\lambda}_{N}^{i} N_{i}$ and $B_{T}^{T} L_{T}=\sum_{i \in I_{C}} T_{i} \tilde{\lambda}_{T}^{i}$. With this notation we obtain a formulation issued from (2.15) involving the nodal forces: for a given $r>0$,

$$
\left\{\begin{array}{l}
\text { find } U \in \mathbb{R}^{k_{1}}, \tilde{L}_{N}=\sum_{i \in I_{C}} \tilde{\lambda}_{N}^{i} N_{i} \text { and } \tilde{L}_{T}=\sum_{i \in I_{C}} T_{i} \tilde{\lambda}_{T}^{i} \text { satisfying } \\
K U=F+\tilde{L}_{N}+\tilde{L}_{T}, \\
\tilde{\lambda}_{N}^{i}=-\left(r u_{N}^{i}-\tilde{\lambda}_{N}^{i}\right)_{+}, \quad 1 \leq i \leq k_{2}, \\
\tilde{\lambda}_{T}^{i}=P_{\bar{B}\left(0,-\mathcal{F} \tilde{\lambda}_{N}^{i}\right)}\left(\tilde{\lambda}_{T}^{i}-r u_{T}^{i}\right), \quad 1 \leq i \leq k_{2},
\end{array}\right.
$$

where $P_{\bar{B}(0, r)}$ is the projection onto the ball of center 0 and radius $r$ in $\mathbb{R}^{d-1}$ and $(x)_{+}$ stands for the positive part of $x \in \mathbb{R}$.

Remark 2.3. The projections in Problem (2.17) are regular unless $\tilde{\lambda}_{N}^{i}-r u_{N}^{i}=0$ or $\left|\tilde{\lambda}_{T}^{i}-r u_{T}^{i}\right|=-\mathcal{F} \tilde{\lambda}_{N}^{i}$. For a solution to Problem (2.17), this is only possible if $\tilde{\lambda}_{N}^{i}=u_{N}^{i}=0$ or $\left|\tilde{\lambda}_{T}^{i}\right|+\mathcal{F} \tilde{\lambda}_{N}^{i}=u_{T}^{i}=0$.

3. The local uniqueness results. Given $r>0$ and denoting $Y=\left(\begin{array}{c}Y_{U} \\ Y_{N} \\ Y_{T}\end{array}\right)$ (with $\left.\left(Y_{U}, Y_{N}, Y_{T}\right) \in \mathbb{R}^{k_{1}} \times \mathbb{R}^{k_{2}} \times \mathbb{R}^{k_{3}}\right)$ and $k=k_{1}+k_{2}+k_{3}$, we consider the Lipschitz-continuous 
and piecewise $\mathcal{C}^{1}$ mapping $\mathcal{G}: \mathbb{R}^{k_{2}} \times \mathbb{R}^{k} \longmapsto \mathbb{R}^{k}$ defined by

$$
\mathcal{G}(G, Y)=\left(\begin{array}{c}
K Y_{U}-F-B_{N}^{T} P_{\bar{\Lambda}_{N}^{h}}\left(Y_{N}-r B_{N} Y_{U}\right)-B_{T}^{T} P_{\bar{\Lambda}_{T}^{h}(G)}\left(Y_{T}-r B_{T} Y_{U}\right) \\
\frac{1}{r}\left(P_{\bar{\Lambda}_{N}^{h}}\left(Y_{N}-r B_{N} Y_{U}\right)-Y_{N}\right) \\
\frac{1}{r}\left(P_{\bar{\Lambda}_{T}^{h}(G)}\left(Y_{T}-r B_{T} Y_{U}\right)-Y_{T}\right)
\end{array}\right) .
$$

The problem

$$
\mathcal{G}(G, Y)=0
$$

corresponds roughly speaking to a friction problem with an a priori known friction threshold $-G$ (also called the Tresca friction problem).

REMARK 3.1. In fact, $\mathcal{G}$ is the gradient of the following proximal augmented Lagrangian introduced by Alart and Curnier [2]:

$$
\begin{aligned}
\mathcal{L}_{r}\left(Y_{U}, Y_{N}, Y_{T}\right)= & \frac{1}{2} Y_{U}^{T} K Y_{U}-Y_{U}^{T} F-Y_{U}^{T} B_{N}^{T} Y_{N}-Y_{U}^{T} B_{T}^{T} Y_{T} \\
& -\frac{1}{2 r}\left\|Y_{N}-r B_{N} Y_{U}-P_{\bar{\Lambda}_{N}^{h}}\left(Y_{N}-r B_{N} Y_{U}\right)\right\|^{2} \\
& -\frac{1}{2 r}\left\|Y_{T}-r B_{T} Y_{U}-P_{\bar{\Lambda}_{T}^{h}(G)}\left(Y_{T}-r B_{T} Y_{U}\right)\right\|^{2} \\
& +\frac{r}{2}\left\|B_{N} Y_{U}\right\|^{2}+\frac{r}{2}\left\|B_{T} Y_{U}\right\|^{2} .
\end{aligned}
$$

In particular, this ensures the symmetry of its gradient.

Now we introduce the map $\mathcal{H}: \mathbb{R} \times \mathbb{R}^{k} \longmapsto \mathbb{R}^{k}$ defined by

$$
\mathcal{H}(\mathcal{F}, Y)=\mathcal{G}\left(\mathcal{F} Y_{N}, Y\right) .
$$

It is straightforward that a solution to Problem (2.15) is a solution to

$$
\mathcal{H}(\mathcal{F}, Y)=0
$$

and conversely. Clearly, $\mathcal{H}=\mathcal{G} \mathcal{I}$ where $\mathcal{I}: \mathbb{R} \times \mathbb{R}^{k} \longmapsto \mathbb{R}^{k_{2}+k}$ is defined by

$$
\mathcal{I}(\mathcal{F}, Y)=\left(\mathcal{F} Y_{N}, Y\right) .
$$

In the following, the notation $\nabla \mathcal{J}=\left(\nabla_{1} \mathcal{J}, \nabla_{2} \mathcal{J}\right)$ represents the Jacobian matrix of a functional $\mathcal{J}(.,$.$) , possibly in a generalized sense (e.g., Clarke's gradient, see [5]).$

Lemma 3.2. Assume that the hypotheses (2.1), (2.2), (2.3) and (2.6) hold and suppose that the mapping $\mathcal{G}$ is $\mathcal{C}^{1}$ near $(G, Y)$. Then the matrix $\nabla_{2} \mathcal{G}(G, Y)$ is nonsingular.

Proof. In this case the Jacobians $\nabla P_{\bar{\Lambda}_{N}^{h}}\left(Y_{N}-r B_{N} Y_{U}\right)$ and $\nabla P_{\bar{\Lambda}_{T}^{h}(G)}\left(Y_{T}-r B_{T} Y_{U}\right)$ exist (in the classical sense) and we denote them by $\nabla P_{\bar{\Lambda}_{N}^{h}}$ and $\nabla P_{\bar{\Lambda}_{T}^{h}(G)}$ for the sake of simplicity. These matrices are symmetric, since a gradient of a projection $P_{C}$ onto a closed convex set $C$ is always symmetric (more precisely $\nabla P_{C}$ is the hessian of $\left(\|x\|^{2}-\right.$ $\left.d_{C}^{2}(x)\right) / 2$ where $d_{C}(x)$ represents the distance from $x$ to $C$ ). To simplify we shall denote $\nabla_{2} \mathcal{G}$ instead of $\nabla_{2} \mathcal{G}(G, Y)$. According to the definition of $\mathcal{G}$ in (3.1) and with the obvious 
notation, we deduce that $\nabla_{2} \mathcal{G}$ is symmetric (the definition of $\mathcal{G}$ was chosen precisely to obtain a symmetric matrix $\nabla_{2} \mathcal{G}$ ) and

$$
\begin{gathered}
\nabla_{2} \mathcal{G}=\left(\begin{array}{ccc}
A_{U U} & A_{U N} & A_{U T} \\
A_{N U} & A_{N N} & 0 \\
A_{T U} & 0 & A_{T T}
\end{array}\right) \\
=\left(\begin{array}{ccc}
K+r B_{N}^{T} \nabla P_{\bar{\Lambda}_{N}^{h}} B_{N}+r B_{T}^{T} \nabla P_{\bar{\Lambda}_{T}^{h}(G)} B_{T} & -B_{N}^{T} \nabla P_{\bar{\Lambda}_{N}^{h}} & -B_{T}^{T} \nabla P_{\bar{\Lambda}_{T}^{h}(G)} \\
-\nabla P_{\bar{\Lambda}_{N}^{h}} B_{N} & \frac{1}{r}\left(\nabla P_{\bar{\Lambda}_{N}^{h}}-I_{2}\right) & 0 \\
-\nabla P_{\bar{\Lambda}_{T}^{h}(G)}^{h} B_{T} & 0 & \frac{1}{r}\left(\nabla P_{\bar{\Lambda}_{T}^{h}(G)}-I_{3}\right)
\end{array}\right)
\end{gathered}
$$

where $I_{2}$ and $I_{3}$ stand for the identity matrices of orders $k_{2}$ and $k_{3}$. So $A_{U U}$ is symmetric and positive definite, and $A_{N N}$ and $A_{T T}$ contain only nonpositive coefficients.

$$
\begin{aligned}
\text { Let }\left(\begin{array}{l}
V_{U} \\
V_{N} \\
V_{T}
\end{array}\right) \neq 0 \text { be such that } \nabla_{2} \mathcal{G}\left(\begin{array}{c}
V_{U} \\
V_{N} \\
V_{T}
\end{array}\right)=0 \text {. Then } \\
\qquad\left(\begin{array}{c}
V_{U} \\
-V_{N} \\
-V_{T}
\end{array}\right)^{T} \nabla_{2} \mathcal{G}\left(\begin{array}{c}
V_{U} \\
V_{N} \\
V_{T}
\end{array}\right)=V_{U}^{T} A_{U U} V_{U}-V_{N}^{T} A_{N N} V_{N}-V_{T}^{T} A_{T T} V_{T}=0,
\end{aligned}
$$

which means that $V_{U}=0$ since $A_{N N}$ and $A_{T T}$ are nonpositive and thus

$$
\begin{aligned}
A_{N N} V_{N} & =0, \\
A_{T T} V_{T} & =0, \\
A_{U N} V_{N}+A_{U T} V_{T} & =0,
\end{aligned}
$$

or equivalently

$$
\begin{aligned}
\left(\nabla P_{\bar{\Lambda}_{N}^{h}}-I_{2}\right) V_{N} & =0, \\
\left(\nabla P_{\bar{\Lambda}_{T}^{h}(G)}-I_{3}\right) V_{T} & =0, \\
-B_{N}^{T} \nabla P_{\bar{\Lambda}_{N}^{h}} V_{N}-B_{T}^{T} \nabla P_{\bar{\Lambda}_{T}^{h}(G)} V_{T} & =0 .
\end{aligned}
$$

Consequently

$$
B_{N}^{T} V_{N}+B_{T}^{T} V_{T}=B^{T}\left(\begin{array}{l}
V_{N} \\
V_{T}
\end{array}\right)=0
$$

Due to the inf-sup condition (2.6) the matrix $B$ has maximal rank, which implies $V_{N}=$ $V_{T}=0$, which contradicts the assumption. Hence $\nabla_{2} \mathcal{G}$ is nonsingular.

The latter result applies when the projections are regular. In fact, most of the time, a solution $Y$ to Problem (3.2) or Problem (3.3) is such that the projections are regular. If $Y_{N}$ lies in the interior of $\bar{\Lambda}_{N}^{h}$, then the projection $P_{\bar{\Lambda}_{N}^{h}}\left(Y_{N}-r B_{N} Y_{U}\right)$ is regular (in fact it reduces then to the identity operator) in a neighborhood of $Y_{N}-r B_{N} Y_{U}$ because necessarily, due to the complementarity condition, one has $B_{N} Y_{U}=0$. Now, if $Y_{N}$ is on the frontier of $\bar{\Lambda}_{N}^{h}$, then very often $Y_{N}-r B_{N} Y_{U}$ will not be in $\bar{\Lambda}_{N}^{h}$ and (depending of course on the particular choice of the set $\bar{\Lambda}_{N}^{h}$ ) most of the time the projection will be regular in a neighborhood of $Y_{N}-r B_{N} Y_{U}$. The situation where the nonregular part of the projection is reached is the case of a "grazing contact": $Y_{N}$ on the frontier of $\bar{\Lambda}_{N}^{h}$ and 
the corresponding part of $B_{N} Y_{U}$ is vanishing. We will study this case in section 4 for a particular discretization. Consider now the case of $Y_{T}$ and suppose that $G=\mathcal{F} Y_{N} \neq 0$ (such a choice of $G$ corresponds to the Coulomb friction case). If $Y_{T}$ lies in the interior of $\bar{\Lambda}_{T}^{h}(G)$, then the projection $P_{\bar{\Lambda}_{T}^{h}(G)}\left(Y_{T}-r B_{T} Y_{U}\right)$ is regular in a neighborhood of $Y_{T}-r B_{T} U$ since $B_{T} Y_{U}=0$. When $Y_{T}$ is on the frontier of $\bar{\Lambda}_{T}^{h}(G)$, then $Y_{T}-r B_{T} Y_{U}$ lies generally outside of $\bar{\Lambda}_{T}^{h}(G)$ and the projection is locally regular. The projection becomes nonregular when the quantity $B_{T} Y_{U}$ is vanishing. This corresponds to a solution which lies at the interface between stick and slip.

Because of the finite-dimensional characteristic of the problem the probability of being in one of the above-mentioned cases is close to zero, but such cases appear and may lead to bifurcations (see the discussion in section 4 ).

Our aim is to study locally the set of solutions verifying (3.3) (or equivalently (2.15)) using implicit function theorems. In the regular case (i.e., when $\mathcal{H}$ is $\mathcal{C}^{1}$ near $(\mathcal{F}, Y)$ ) we have to consider the $k$-by- $k$ matrix $\nabla_{2} \mathcal{H}$ containing the derivatives of $\mathcal{H}$ with respect to $\left(Y_{U}, Y_{N}, Y_{T}\right)$ and to discuss its singularity. Consequently

$$
\begin{aligned}
\nabla_{2} \mathcal{H}(\mathcal{F}, Y) & =\nabla \mathcal{G}\left(\mathcal{F} Y_{N}, Y\right) \nabla_{2} \mathcal{I}(\mathcal{F}, Y) \\
& =\nabla_{1} \mathcal{G}\left(\mathcal{F} Y_{N}, Y\right)\left(0_{1} \mathcal{F} I_{2} 0_{3}\right)+\nabla_{2} \mathcal{G}\left(\mathcal{F} Y_{N}, Y\right)
\end{aligned}
$$

where $0_{1}\left(\right.$ resp. $\left.0_{3}\right)$ denotes the $k_{2}$-by- $k_{1}\left(\right.$ resp. $k_{2}$-by- $\left.k_{3}\right)$ zero matrix and $I_{2}$ represents the identity matrix of order $k_{2}$.

We look for conditions ensuring singularity of $\nabla_{2} \mathcal{H}=\nabla_{2} \mathcal{H}(\mathcal{F}, Y)$. Assume that $\nabla_{2} \mathcal{H}(\mathcal{F}, Y)$ is singular. According to Lemma 3.2 the matrix $\nabla_{2} \mathcal{G}=\nabla_{2} \mathcal{G}\left(\mathcal{F} Y_{N}, Y\right)$ is nonsingular and we write $\nabla_{1} \mathcal{G}$ instead of $\nabla_{1} \mathcal{G}\left(\mathcal{F} Y_{N}, Y\right)$ to simplify. From (3.4) there exists a nonzero $\left(V_{U} V_{N} V_{T}\right)$ such that

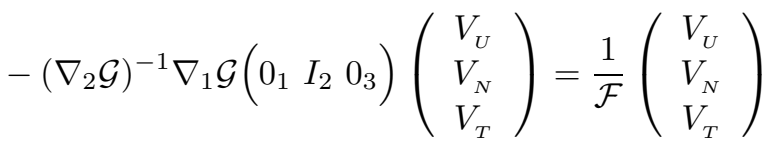

or equivalently

$$
-\left(\nabla_{2} \mathcal{G}\right)^{-1} \nabla_{1} \mathcal{G} V_{N}=\frac{1}{\mathcal{F}}\left(\begin{array}{c}
V_{U} \\
V_{N} \\
V_{T}
\end{array}\right) .
$$

In fact it suffices to determine an eigenpair $\left(\frac{1}{\mathcal{F}}, V_{N}\right)$ such that

$$
A V_{N}=\frac{1}{\mathcal{F}} V_{N},
$$

where $A$ is the matrix obtained from $-\left(\nabla_{2} \mathcal{G}\right)^{-1} \nabla_{1} \mathcal{G}$ when keeping the lines from $k_{1}+1$ to $k_{1}+k_{2}$ (in other words $\left.A=-\left(\begin{array}{lll}0_{1} & I_{2} & 0_{3}\end{array}\right)\left(\nabla_{2} \mathcal{G}\right)^{-1} \nabla_{1} \mathcal{G}\right)$. Clearly, $A$ is of order $k_{2}$ and Problem (3.7) admits exactly $k_{2}$ eigenvalues (which depend on the contact status $(\mathcal{F}, Y))$. Besides, note that if $\left(1 / \mathcal{F}, V_{N}\right)$ is given by the eigenvalue problem (3.7), then $V_{U}$ and $V_{T}$ can be obtained from (3.6). 
Using the standard implicit function theorem we can state the following local uniqueness result.

Proposition 3.3. Assume that the hypotheses (2.1), (2.2), (2.3) and (2.6) hold. If $\left(\mathcal{F}_{0}, Y_{0}\right)$ solves (3.3), if $\mathcal{H}$ is $\mathcal{C}^{1}$ near $\left(\mathcal{F}_{0}, Y_{0}\right)$ and if $\frac{1}{\mathcal{F}_{0}}$ is not an eigenvalue to Problem (3.7), then there exist a neighborhood $U$ of $\mathcal{F}_{0}$, a neighborhood $V$ of $Y_{0}$ and a $\mathcal{C}^{1}$ function $\zeta: U \longrightarrow V$ such that

$$
\mathcal{H}(\mathcal{F}, Y)=0, \mathcal{F} \in U, Y \in V \Longleftrightarrow Y=\zeta(\mathcal{F})
$$

Since the situation is clear in the regular setting, we now consider the nonregular case. Because the maps $\mathcal{G}$ and $\mathcal{H}$ are Lipschitz continuous, they admit a generalized gradient in Clarke's sense everywhere. Let us recall the following characterization of the generalized gradient (see [5]) for a Lipschitz-continuous function $\mathcal{J}(Z)$ which fails to be differentiable in a set $\Omega_{f}$ of zero Lebesgue measure:

$$
\nabla \mathcal{J}(Z)=\operatorname{co}\left\{\lim _{i \rightarrow \infty} \nabla \mathcal{J}\left(Z_{i}\right): Z_{i} \rightarrow Z, Z_{i} \notin \Omega_{f}\right\},
$$

where the notation "co" stands for the convex hull (see [17]).

The computation of the generalized gradient of $\mathcal{G}$ leads to the following result:

$$
\begin{gathered}
\nabla \mathcal{G}(G, Y)=\left(\begin{array}{cccc}
A_{U G} & A_{U U} & A_{U N} & A_{U T} \\
0 & A_{N U} & A_{N N} & 0 \\
A_{T G} & A_{T U} & 0 & A_{T T}
\end{array}\right) \\
=\left(\begin{array}{cccc}
-B_{T}^{T} W_{G} & K+r B_{N}^{T} W_{N} B_{N}+r B_{T}^{T} W_{T} B_{T} & -B_{N}^{T} W_{N} & -B_{T}^{T} W_{T} \\
0 & -W_{N} B_{N} & \frac{1}{r}\left(W_{N}-I_{2}\right) & 0 \\
\frac{1}{r} W_{G} & -W_{T} B_{T} & 0 & \frac{1}{r}\left(W_{T}-I_{3}\right)
\end{array}\right)
\end{gathered}
$$

where $W_{N}$ and $\left(W_{T} W_{G}\right)$ are selections of the generalized gradient of $X \mapsto P_{\bar{\Lambda}_{N}^{h}}(X)$ taken at $Y_{N}-r B_{N} Y_{U}$ and of the generalized gradient of $(X, G) \mapsto P_{\bar{\Lambda}_{T}^{h}(G)}(X)$ taken at $Y_{T}-r B_{T} Y_{U}$ respectively. The matrices $W_{N}$ and $W_{T}$ are symmetric because of the symmetry of the gradient of a projection.

The generalized gradient of $\mathcal{H}$ can be deduced:

$$
\nabla \mathcal{H}(\mathcal{F}, Y)=\left(\begin{array}{cccc}
-B_{T}^{T} W_{G} Y_{N} & A_{U U} & A_{U N} & A_{U T} \\
0 & A_{N U} & A_{N N} & 0 \\
\frac{1}{r} W_{G} Y_{N} & A_{T U} & 0 & A_{T T}
\end{array}\right)+\left(\begin{array}{c}
A_{U G} \\
0 \\
A_{T G}
\end{array}\right)\left(\begin{array}{llll}
0 & 0_{1} & \mathcal{F} I_{2} & 0_{3}
\end{array}\right),
$$

and its restriction to the second variable becomes

$$
\nabla_{2} \mathcal{H}(\mathcal{F}, Y)=\left(\begin{array}{ccc}
A_{U U} & A_{U N} & A_{U T} \\
A_{N U} & A_{N N} & 0 \\
A_{T U} & 0 & A_{T T}
\end{array}\right)+\left(\begin{array}{c}
A_{U G} \\
0 \\
A_{T G}
\end{array}\right)\left(\begin{array}{lll}
0_{1} & \mathcal{F} I_{2} & 0_{3}
\end{array}\right) .
$$

Note that in the case of generalized gradients, the right way to compute a partial gradient is to restrict the total gradient; see [5]. In particular, $W_{G}$ and $W_{T}$ are not independent selections. The following lemma is the extension to the nonregular case of Lemma 3.2 . 
Lemma 3.4. Assume that the hypotheses (2.1), (2.2), (2.3), (2.6) and (2.16) hold. Then the restriction $\nabla_{2} \mathcal{G}(G, Y)$ of the generalized gradient $\nabla \mathcal{G}(G, Y)$ is nonsingular in the sense that any selection of $\nabla_{2} \mathcal{G}(G, Y)$ is a nonsingular matrix.

Proof. The proof of this lemma is identical to the one of Lemma 3.2 applied to each selection of $\nabla_{2} \mathcal{G}(G, Y)$.

It is straightforward that $\nabla_{2} \mathcal{H}(\mathcal{F}, Y)$ is singular if and only if there exist $\left(\begin{array}{c}V_{U} \\ V_{N} \\ V_{T}\end{array}\right) \neq 0$ and a selection of $W_{N}$ and $\left(W_{G} W_{T}\right)$ such that

$$
\left(\begin{array}{ccc}
A_{U U} & A_{U N} & A_{U T} \\
A_{N U} & A_{N N} & 0 \\
A_{T U} & 0 & A_{T T}
\end{array}\right)\left(\begin{array}{c}
V_{U} \\
V_{N} \\
V_{T}
\end{array}\right)+\mathcal{F}\left(\begin{array}{c}
A_{U G} \\
0 \\
A_{T G}
\end{array}\right) V_{N}=0
$$

i.e., there exists an eigenpair $\left(\frac{1}{\mathcal{F}}, V_{N}\right)$ such that

$$
-\left(\begin{array}{lll}
0_{1} & I_{2} & 0_{3}
\end{array}\right)\left(\begin{array}{ccc}
A_{U U} & A_{U N} & A_{U T} \\
A_{N U} & A_{N N} & 0 \\
A_{T U} & 0 & A_{T T}
\end{array}\right)^{-1}\left(\begin{array}{c}
A_{U G} \\
0 \\
A_{T G}
\end{array}\right) V_{N}=\frac{1}{\mathcal{F}} V_{N},
$$

which is the same formulation as in (3.6) (or (3.7)) with generalized gradients. Thus, the following theorem holds:

Theorem 3.5. Assume that the hypotheses (2.1), (2.2), (2.3), (2.6) and (2.16) hold. If $\left(\mathcal{F}_{0}, Y_{0}\right)$ solves (3.3) and if $\frac{1}{\mathcal{F}_{0}}$ is not an eigenvalue to Problem (3.8) for any selection of $W_{N}, W_{G}$ and $W_{T}$, then there exist a neighborhood $U$ of $\mathcal{F}_{0}$, a neighborhood $V$ of $Y_{0}$ and a Lipschitz-continuous function $\zeta: U \longrightarrow V$ such that

$$
\mathcal{H}(\mathcal{F}, Y)=0, \mathcal{F} \in U, Y \in V \Longleftrightarrow Y=\zeta(\mathcal{F}) .
$$

The proof is a straightforward application of the implicit function theorem for Lipschitz functions due to J.-B. Hiriart-Urruty (See [16] or [5]).

Note that the result in the theorem (as well as the one in the proposition) is only a sufficient condition for local uniqueness and continuation of solutions. These conditions are not necessary in the general case (see the discussion at the end of section 4).

4. An elementary example. We now discuss an elementary example involving the single linear triangular finite element in two space dimensions depicted in Figure 2 (the lengths of $\Gamma_{C}, \Gamma_{N}$ and $\Gamma_{D}$ are $\ell, \ell$ and $\sqrt{2} \ell$, respectively). We use Hooke's constitutive law corresponding to homogeneous isotropic materials in (1.2):

$$
\sigma_{i j}(u)=\lambda \delta_{i j} \varepsilon_{k k}(u)+2 \mu \varepsilon_{i j}(u) \quad \text { in } \Omega,
$$

where $\lambda \geq 0$ and $\mu>0$ are the Lamé coefficients and $\delta_{i j}$ denotes the Kronecker symbol. Note that in plane strain approximation $\lambda=(E \nu) /((1-2 \nu)(1+\nu))$ and $\mu=E /(2(1+$ $\nu)$ ) where $E>0$ and $0 \leq \nu<1 / 2$ represent Young's modulus and Poisson's ratio, respectively. 


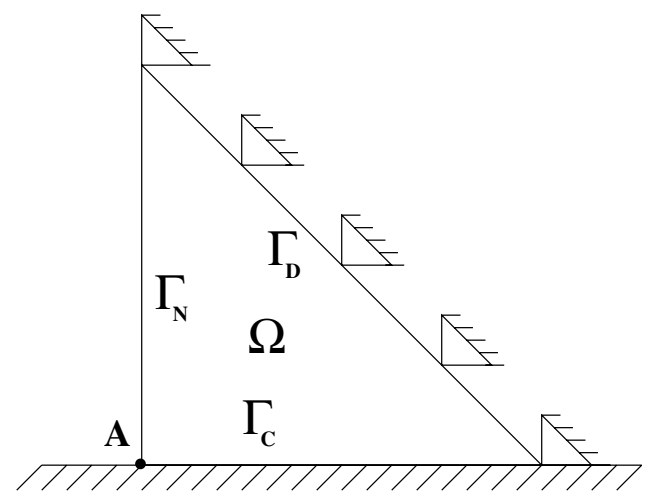

FiG. 2. Example of an elementary finite element mesh

Our aim in this section is to achieve a complete discussion concerning local uniqueness and continuation of solutions for this simple finite element problem. We solve the eigenvalue problem (3.7) and the nonsmooth version (3.8) and we compare the results given by the proposition and the theorem with the set of solutions to (3.3). In this configuration the contact status is only determined by the behavior of point $A$. We adopt the finite element discretisation of the frictional contact conditions written in (2.17). As a consequence we have $\left(k_{1}, k_{2}, k_{3}\right)=(2,1,1)$; therefore for a given friction coefficient and a loading there are four unknowns to the frictional contact problem. We denote them by $u_{N}$ (normal displacement at $A$ ), $u_{T}$ (tangential displacement at $A$ ), $\tilde{\lambda}_{N}$ (normal component of the generalized load at $A$ ) and $\tilde{\lambda}_{T}$ (tangential component of the generalized load at $A)$. On $\Gamma_{C}$ we set $\mathbf{n}=(0,-1)$ and the unit tangent vector is $\mathbf{t}=(1,0)$. The matrices $K, B_{N}, B_{T}$ become:

$$
K=\frac{1}{2}\left(\begin{array}{cc}
\lambda+3 \mu & -(\lambda+\mu) \\
-(\lambda+\mu) & \lambda+3 \mu
\end{array}\right) \quad B_{N}=\left(\begin{array}{ll}
\ell / 3 & 0
\end{array}\right) \quad B_{T}=\left(\begin{array}{ll}
0 & \ell / 3
\end{array}\right) .
$$

With $F^{T}=\left(F_{N}, F_{T}\right)$, and according to (2.17) the discrete frictional contact problem is:

$$
\left\{\begin{array}{l}
\frac{(\lambda+3 \mu)}{2} u_{N}-\frac{(\lambda+\mu)}{2} u_{T}=\tilde{\lambda}_{N}+F_{N}, \\
-\frac{(\lambda+\mu)}{2} u_{N}+\frac{(\lambda+3 \mu)}{2} u_{T}=\tilde{\lambda}_{T}+F_{T}, \\
\tilde{\lambda}_{N}=P_{]-\infty, 0]}\left(\tilde{\lambda}_{N}-r u_{N}\right), \\
\tilde{\lambda}_{T}=P_{\left[\mathcal{F} \tilde{\lambda}_{N},-\mathcal{F} \tilde{\lambda}_{N}\right]}\left(\tilde{\lambda}_{T}-r u_{T}\right) .
\end{array}\right.
$$

Let us first carry out a simple calculation, which is useful for the forthcoming study. Consider the mapping $T: \mathbb{R} \times \mathbb{R}_{+} \rightarrow \mathbb{R}$ such that

$$
T(x, y)=P_{[-y, y]}(x) .
$$

It is easy to see that $T$ is Lipschitz continuous and differentiable in Clarke's sense. Its gradient is given in Fig. 3. Note that for $x>0$, one has $\nabla_{x} T(x, x)=\alpha \in[0,1]$ and 


$$
\begin{aligned}
& \begin{array}{l}
\nabla T=\alpha\left(\begin{array}{l}
1 \\
0
\end{array}\right)+(1-\alpha)\left(\begin{array}{c}
0 \\
-1
\end{array}\right) \\
(0>x=-y, 0 \leq \alpha \leq 1)
\end{array} \\
& \begin{array}{l}
\nabla T=\left(\begin{array}{c}
0 \\
-1
\end{array}\right) \\
(x<-y)
\end{array} \\
& \left(x=y=0,0 \leq\left(\begin{array}{l}
1 \\
0
\end{array}\right)+\beta\left(\begin{array}{l}
0 \\
1
\end{array}\right)\right. \\
& \begin{array}{l}
\nabla T=1,|\beta| \\
(-y \nmid 1-\alpha)
\end{array}
\end{aligned}
$$

FIG. 3. Generalized gradient of the map $T(x, y)=P_{[-y, y]}(x)$

$\nabla_{y} T(x, x)=1-\alpha$ and the two partial derivatives are dependent.

Now, from (3.8) and Theorem 3.5, a solution $(\mathcal{F}, Y)$ is locally unique provided that for any selection $W_{N} \in \nabla P_{-\infty, 0]}\left(\tilde{\lambda}_{N}-r u_{N}\right)$ and $\left(W_{T}-W_{G}\right) \in \nabla T\left(\tilde{\lambda}_{T}-r u_{T},-\mathcal{F} \tilde{\lambda}_{N}\right)$, there is no nonzero solution $V=\left(\begin{array}{c}V_{U N} \\ V_{U T} \\ V_{N} \\ V_{T}\end{array}\right)$ to the following system:

$$
\begin{array}{r}
\left(\begin{array}{cccc}
\frac{\lambda+3 \mu}{2}+\frac{r \ell^{2}}{9} W_{N} & -\frac{\lambda+\mu}{2} & -\frac{\ell}{3} W_{N} & 0 \\
-\frac{\lambda+\mu}{2} & \frac{\lambda+3 \mu}{2}+\frac{r \ell^{2}}{9} W_{T} & 0 & -\frac{\ell}{3} W_{T} \\
-\frac{\ell}{3} W_{N} & 0 & \frac{1}{r}\left(W_{N}-1\right) & 0 \\
0 & -\frac{\ell}{3} W_{T} & 0 & \frac{1}{r}\left(W_{T}-1\right)
\end{array}\right)\left(\begin{array}{c}
V_{U N} \\
V_{U T} \\
V_{N} \\
V_{T}
\end{array}\right) \\
0 \\
\quad+\mathcal{F}\left(\begin{array}{c}
0 \\
-\frac{\ell}{3} W_{G} \\
0 \\
\frac{1}{r} W_{G}
\end{array}\right) V_{N}=0 .
\end{array}
$$

4.1. Analysis of the different cases. The different situations are summarized in Table 1 .

Cases a1 and a2 deal with nonzero contact forces located on the boundary of Coulomb's cone. Case a1 corresponds to contact with slip and the solution is locally unique if $u_{T}>0$. The solution is also locally unique if $u_{T}<0$ and $\mathcal{F} \neq(\lambda+3 \mu) /(\lambda+\mu)$. The remaining case (i.e., $u_{T}<0$ and $\left.\mathcal{F}=(\lambda+3 \mu) /(\lambda+\mu)\right)$ may correspond to a bifurcation case, and it will be analyzed in section 4.3. In case a2 there is sticking contact, and the solution is locally unique when $\tilde{\lambda}_{T}<0$. The solution is also unique when $\tilde{\lambda}_{T}>0$ and $\mathcal{F} \in\left[0,(\lambda+3 \mu) /(\lambda+\mu)\left[\right.\right.$. Bifurcation may occur for $\mathcal{F} \geq(\lambda+3 \mu) /(\lambda+\mu)$ and $\tilde{\lambda}_{T}>0$.

Case a3 corresponds to contact forces in the interior of Coulomb's cone, and case a4 corresponds to a noncontact state. In both cases the solution is locally unique for any positive friction coefficient. 


\begin{tabular}{|c|c|c|c|}
\hline Cases & $\begin{array}{l}\text { Values of } W_{N} \\
W_{T} \text { and } W_{G}\end{array}$ & $\begin{array}{l}\text { Values of } \mathcal{F} \text { for which system } \\
\text { (4.2) has nontrivial solutions }\end{array}$ & $\begin{array}{l}\text { Range of } \mathcal{F} \\
\text { and condi- } \\
\text { tion }\end{array}$ \\
\hline $\begin{array}{l}\text { a1) } u_{N}=0 \\
\tilde{\lambda}_{T}= \pm \mathcal{F} \tilde{\lambda}_{N} \\
\quad \neq 0 \\
\text { and } u_{T} \neq 0\end{array}$ & $\begin{array}{l}W_{N}=1, W_{T}=0 \\
W_{G}=\operatorname{sgn}\left(u_{T}\right)\end{array}$ & $-\operatorname{sgn}\left(u_{T}\right) \frac{a}{b}$ & $\begin{array}{l}\mathcal{F}=\frac{a}{b} \\
u_{T}<0\end{array}$ \\
\hline $\begin{array}{l}\text { a2) } u_{N}=0 \\
\tilde{\lambda}_{T}= \pm \mathcal{F} \tilde{\lambda}_{N} \\
\quad \neq 0 \\
\text { and } u_{T}=0\end{array}$ & $\begin{array}{l}W_{N}=1, \\
W_{T}=\alpha \in[0,1], \\
W_{G}=-\operatorname{sgn}\left(\tilde{\lambda}_{T}\right)(1-\alpha)\end{array}$ & $\operatorname{sgn}\left(\tilde{\lambda}_{T}\right)\left(\frac{a}{b}+\frac{r \ell^{2}}{9 b} \frac{\alpha}{1-\alpha}\right)$ & $\begin{array}{l}\mathcal{F} \in\left[\frac{a}{b},+\infty[,\right. \\
\tilde{\lambda}_{T}>0\end{array}$ \\
\hline $\begin{array}{l}\text { a3) } \\
\left|\tilde{\lambda}_{T}\right|<-\mathcal{F} \tilde{\lambda}_{N}\end{array}$ & $\begin{array}{l}W_{N}=1, W_{T}=1 \\
W_{G}=0\end{array}$ & none & $\emptyset$ \\
\hline $\begin{array}{l}\text { a4) } u_{N}<0 \text { and } \\
\tilde{\lambda}_{T}=\tilde{\lambda}_{N}=0\end{array}$ & $W_{N}=0\left(\right.$ so $\left.V_{N}=0\right)$ & none & $\emptyset$ \\
\hline $\begin{array}{l}\text { a5) } u_{N}=0 \\
\tilde{\lambda}_{T}=\tilde{\lambda}_{N}=0 \\
\text { and } u_{T} \neq 0\end{array}$ & $\begin{array}{l}W_{N}=\gamma \in[0,1] \\
W_{T}=0 \\
W_{G}=\operatorname{sgn}\left(u_{T}\right)\end{array}$ & $-\operatorname{sgn}\left(u_{T}\right)\left(\frac{a}{b}+\frac{1-\gamma}{\gamma} \frac{9\left(a^{2}-b^{2}\right)}{r \ell^{2} b}\right)$ & $\begin{array}{l}\mathcal{F} \in\left[\frac{a}{b},+\infty[\right. \\
u_{T}<0\end{array}$ \\
\hline $\begin{array}{l}\text { a6) } u_{N}=0 \\
\tilde{\lambda}_{T}=\tilde{\lambda}_{N}=0 \\
\text { and } u_{T}=0\end{array}$ & $\begin{array}{l}W_{N}=\gamma \in[0,1] \\
W_{T}=\alpha \in[0,1] \\
W_{G}=\beta \in[\alpha-1,1-\alpha]\end{array}$ & $\begin{array}{l}\frac{9}{r \beta \ell^{2}}\left(\frac{b(1-\alpha)(1-\gamma)}{\gamma}-\right. \\
\left.\left(\frac{a(1-\alpha)}{b}+\frac{r \alpha \ell^{2}}{9 b}\right)\left(\frac{a(1-\gamma)}{\gamma}+\frac{r \ell^{2}}{9}\right)\right)\end{array}$ & $\mathcal{F} \in\left[\frac{a}{b},+\infty[\right.$ \\
\hline
\end{tabular}

TABLE 1. Cases of nontrivial solutions for system (4.2) with $a=$ $(\lambda+3 \mu) / 2$ and $b=(\lambda+\mu) / 2$.

Cases a5 and a6 correspond to vanishing contact forces (e.g., grazing contact). The solution is locally unique for $u_{T}>0$ or for $u_{T} \leq 0$ with $\mathcal{F} \in[0,(\lambda+3 \mu) /(\lambda+\mu)[$ (in case a6 we note that the friction coefficient value $a / b$ is obtained when $\alpha=0, \beta=-1, \gamma=1)$. Here also, bifurcations may be possible in the case $u_{T} \leq 0$ and $\mathcal{F} \geq(\lambda+3 \mu) /(\lambda+\mu)$.

4.2. Solutions to the elementary example. It is not difficult to obtain all the solutions to Problem (4.1) for any positive friction coefficient $\mathcal{F}$. They are listed in Table 2

Combining the results of the previous discussion we come to the conclusion that there are four situations in which more than one solution exists. More precisely the problem admits:

- an infinity of solutions coming from case b6 as well as the solutions of cases b1 and b2, when $0<(\lambda+3 \mu) F_{N}=-(\lambda+\mu) F_{T}$ and $\mathcal{F}=\frac{(\lambda+3 \mu)}{(\lambda+\mu)}$;

- three solutions coming from the cases b1, b2 and b5 for $F_{N}>0, \mathcal{F}>\frac{(\lambda+3 \mu)}{(\lambda+\mu)}$ and $-\mathcal{F} F_{N}<F_{T}<-\frac{(\lambda+3 \mu)}{(\lambda+\mu)} F_{N}$ 


\begin{tabular}{|c|c|c|}
\hline Cases & Solutions & Range of $F_{N}$ and $F_{T}$ \\
\hline $\begin{array}{l}\mathrm{b} 1) \\
\text { no contact forces } \\
\tilde{\lambda}_{N}=0\end{array}$ & $\begin{array}{l}\tilde{\lambda}_{N}=\tilde{\lambda}_{T}=0 \\
u_{N}=\frac{a F_{N}+b F_{T}}{a^{2}-b^{2}}, \\
u_{T}=\frac{b F_{N}+a F_{T}}{a^{2}-b^{2}}\end{array}$ & $a F_{N}+b F_{T} \leq 0$ \\
\hline $\begin{array}{l}\text { b2) stick contact } \\
u_{T}=0, \\
\left|\tilde{\lambda}_{T}\right| \leq-\mathcal{F} \tilde{\lambda}_{N} \neq 0\end{array}$ & $\begin{array}{l}u_{N}=u_{T}=0, \tilde{\lambda}_{N}=-F_{N} \\
\tilde{\lambda}_{T}=-F_{T}\end{array}$ & $\begin{array}{l}F_{N}>0 \\
-\mathcal{F} F_{N} \leq F_{T} \leq \mathcal{F} F_{N}\end{array}$ \\
\hline $\begin{array}{l}\text { b3) } \quad \text { slip contact } \\
u_{T}>0, \\
\tilde{\lambda}_{T}=\mathcal{F} \tilde{\lambda}_{N} \neq 0\end{array}$ & $\begin{array}{l}u_{N}=0, \tilde{\lambda}_{T}=\mathcal{F} \tilde{\lambda}_{N}, \\
\tilde{\lambda}_{N}=-\frac{a F_{N}+b F_{T}}{a+b \mathcal{F}}, \\
u_{T}=\frac{F_{T}-\mathcal{F} F_{N}}{a+b \mathcal{F}}\end{array}$ & $\begin{array}{l}a F_{N}+b F_{T}>0 \\
F_{T}-\mathcal{F} F_{N}>0\end{array}$ \\
\hline $\begin{array}{l}\text { b4) slip contact } \\
u_{T}<0 \\
\tilde{\lambda}_{T}=-\mathcal{F} \tilde{\lambda}_{N} \neq 0 \\
\text { and } \mathcal{F}<\frac{a}{b}\end{array}$ & $\begin{array}{l}u_{N}=0, \tilde{\lambda}_{T}=-\mathcal{F} \tilde{\lambda}_{N} \\
\tilde{\lambda}_{N}=-\frac{a F_{N}+b F_{T}}{a-b \mathcal{F}} \\
u_{T}=\frac{F_{T}+\mathcal{F} F_{N}}{a-b \mathcal{F}}\end{array}$ & $\begin{array}{l}a F_{N}+b F_{T}>0 \\
F_{T}+\mathcal{F} F_{N}<0\end{array}$ \\
\hline $\begin{array}{l}\text { b5) slip contact } \\
u_{T}<0, \\
\tilde{\lambda}_{T}=-\mathcal{F} \tilde{\lambda}_{N} \neq 0 \\
\text { and } \mathcal{F}>\frac{a}{b}\end{array}$ & $\begin{array}{l}u_{N}=0, \tilde{\lambda}_{T}=-\mathcal{F} \tilde{\lambda}_{N} \\
\tilde{\lambda}_{N}=-\frac{a F_{N}+b F_{T}}{a-b \mathcal{F}} \\
u_{T}=\frac{F_{T}+\mathcal{F} F_{N}}{a-b \mathcal{F}}\end{array}$ & $\begin{array}{l}a F_{N}+b F_{T}<0 \\
F_{T}+\mathcal{F} F_{N}>0\end{array}$ \\
\hline $\begin{array}{l}\text { b6) } \quad \text { slip contact } \\
u_{T}<0, \\
\tilde{\lambda}_{T}=-\mathcal{F} \tilde{\lambda}_{N} \neq 0 \\
\text { and } \mathcal{F}=\frac{a}{b}\end{array}$ & $\begin{array}{l}u_{N}=0, \tilde{\lambda}_{T}=-\mathcal{F} \tilde{\lambda}_{N} \\
\frac{-F_{N}}{b}<u_{T}<0 \\
\tilde{\lambda}_{N}=-\left(F_{N}+b u_{T}\right) \\
\text { (infinitely many solutions) }\end{array}$ & $a F_{N}=-b F_{T}, F_{N}>0$ \\
\hline
\end{tabular}

TABLE 2. All the solutions to the elementary example with $a=$ $(\lambda+3 \mu) / 2$ and $b=(\lambda+\mu) / 2$

- two solutions coming from the cases b1 and b2 when $F_{N}>0, \mathcal{F}>\frac{(\lambda+3 \mu)}{(\lambda+\mu)}$ and $-\mathcal{F} F_{N}<F_{T}=-\frac{(\lambda+3 \mu)}{(\lambda+\mu)} F_{N}$

- two solutions coming from the cases b1 and b2 when $F_{N}>0, \mathcal{F}>\frac{(\lambda+3 \mu)}{(\lambda+\mu)}$ and $-\mathcal{F} F_{N}=F_{T}<-\frac{(\lambda+3 \mu)}{(\lambda+\mu)} F_{N}$.

4.3. Bifurcation diagrams. Analyzing the solutions given in Table 2, we conclude that for $(\lambda+3 \mu) F_{N}+(\lambda+\mu) F_{T}>0$ the solution to Problem (4.1) is unique for any friction 


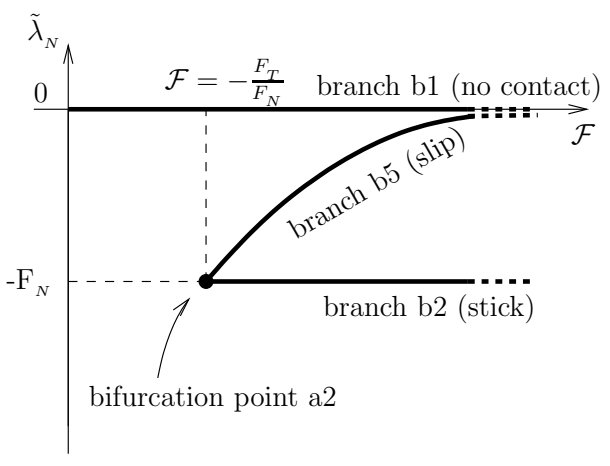

Bifurcation diagram $\left(\tilde{\lambda}_{N}, \mathcal{F}\right)$

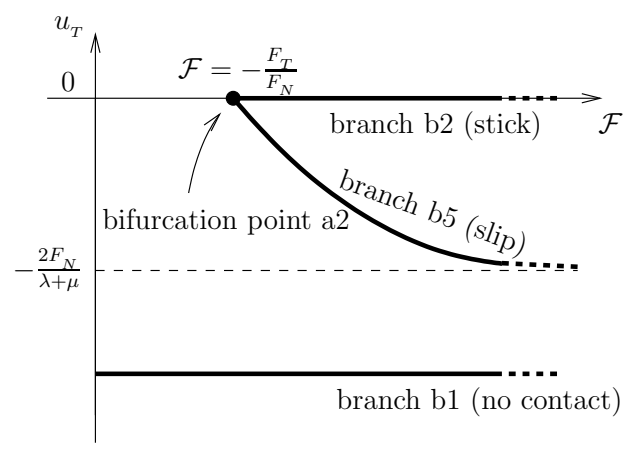

Bifurcation diagram $\left(u_{T}, \mathcal{F}\right)$

FIG. 4. First case of bifurcation

coefficient. This is also the case when $(\lambda+3 \mu) F_{N}+(\lambda+\mu) F_{T} \leq 0$ and $F_{N} \leq 0$. In fact there are only two loading situations where a (global) multiplicity of solutions occurs: the first case is $(\lambda+3 \mu) F_{N}+(\lambda+\mu) F_{T}<0$ and $F_{N}>0$ and the second (more particular) case is $(\lambda+3 \mu) F_{N}+(\lambda+\mu) F_{T}=0$ and $F_{N}>0$.

4.3.1. Case $(\lambda+3 \mu) F_{N}+(\lambda+\mu) F_{T}<0$ and $F_{N}>0$. In this case, the solution b1 of Table 2 is valid for any friction coefficient. This solution corresponds to a separation of point $A$ from the rigid foundation. Moreover the solution given by $\mathrm{b} 2$ solves the problem for any $\mathcal{F} \geq-F_{T} / F_{N}$ whereas case b5 is valid for $\mathcal{F}>-F_{T} / F_{N}$. The two branches b2 and b5 intersect each other at $\mathcal{F}=-F_{T} / F_{N}$ in a bifurcation point of type a2 relative to the classification of Table 1, A very interesting characteristic is that the bifurcation diagram is not connected and there is no bifurcation from the branch b1 (see Fig. 4).

4.3.2. Case $(\lambda+3 \mu) F_{N}+(\lambda+\mu) F_{T}=0$ and $F_{N}>0$. In this case the solution b1 of Table 2 is valid for any friction coefficient. We observe that this solution represents grazing contact with nonzero slip. The solution given by b2 is valid for $\mathcal{F} \geq(\lambda+3 \mu) /$ $(\lambda+\mu)$ and solution b6 is valid for $\mathcal{F}=(\lambda+3 \mu) /(\lambda+\mu)$. In fact, it can be viewed as the limit case of the bifurcation diagram considered previously when $(\lambda+3 \mu) F_{N}+(\lambda+\mu) F_{T}$ goes to zero with negative values. Here the diagram is connected and it contains the vertical branch b6. The bifurcation points are of type a1, a2 and a5 following the classification of Table 1 (see Fig. 5).

REMARK 4.1. For a solution of case b1 corresponding to grazing contact with nonzero slip, the friction coefficient is an eigenvalue when $\mathcal{F} \geq(\lambda+3 \mu) /(\lambda+\mu)$ (case a5 of Table 11). The absence of bifurcation for $\mathcal{F}>(\lambda+3 \mu) /(\lambda+\mu)$ illustrates the fact that the assumption leading to the local uniqueness and continuation result of Theorem 3.5 is a sufficient condition which is not necessary. This is also the case when $F_{N}=F_{T}=0$ where $u_{N}=u_{N}=\tilde{\lambda}_{N}=\tilde{\lambda}_{T}=0$ is the unique solution for any friction coefficient although this is a point of type a6. 


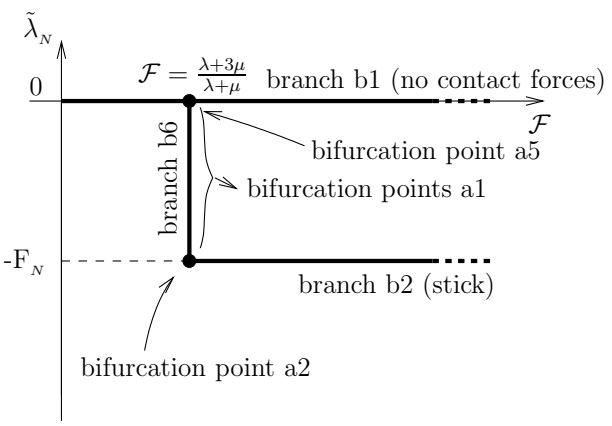

Bifurcation diagram $\left(\tilde{\lambda}_{N}, \mathcal{F}\right)$

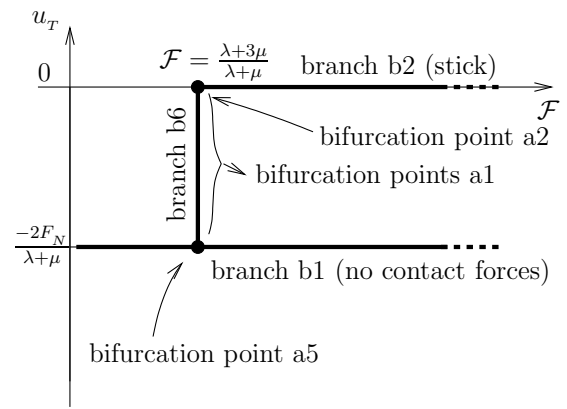

Bifurcation diagram $\left(u_{T}, \mathcal{F}\right)$

FIG. 5. Second case of bifurcation

5. Concluding remarks. This work is a first contribution dealing with local uniqueness and continuation of solutions without any smallness assumption on the friction coefficient. We prove that the solutions are locally unique if the friction coefficient does not belong to the spectrum of a contact status depending matrix. We discuss in detail a simple case for which we exhibit some nonsmooth bifurcation diagrams, one of them being not connected.

Nevertheless several questions concerning this study remain open. An interesting study would be to classify the bifurcations which occur when the friction coefficient reaches an eigenvalue and to give necessary and sufficient conditions for a bifurcation to appear. Of course, this should be rather more complex in this nonsmooth context compared to the classical smooth one presented for instance in [25]. Another line of research would be to extend these results to the continuum case.

Although the analysis we made is dedicated to the static case, the latter is very close to the problem coming from a time step of an implicit scheme of a dynamic or quasistatic friction problem. For instance, in the dynamical case, the discretized problem coming from a time step is merely the same as Problem (2.15) when adding a source term coming from the previous time step, changing $K U$ with $M /(\Delta t)^{2}+K U$ and $B_{T} U$ with $B_{T} V$ where $\Delta t$ is the time step, $M$ is the mass matrix and $V$ is the discretization of the velocity. So our work can be trivially extended to this case showing some bifurcations of the fully discretized scheme.

From the term $M /(\Delta t)^{2}$ one could believe that for a sufficiently small time step $\Delta t$ the bifurcations disappear. It seems that this is not the case, due to the term $B_{T} V$ in the friction condition which implicitly contains a $1 / \Delta t$ factor. An interesting open problem would be to study the evolution of the bifurcations (i.e., the evolution of the corresponding eigenvalue problems) when $h$ and $\Delta t$ tend to zero. For the moment, no evidence of dynamic bifurcations has been exhibited for the continuous dynamical friction problem except when the friction coefficient is a decreasing function of the sliding velocity (see [18] and [26] for instance). 


\section{REFERENCES}

[1] R.A. Adams, Sobolev spaces, Academic Press, 1975. MR0450957 (56:9247)

[2] A. Alart, A. Curnier, A mixed formulation for frictional contact problems prone to Newton like solution methods. Comput. Methods Appl. Mech. Engrg. 92 (1991), 353-375. MR1141048 (92i:73087)

[3] L.-E. Andersson, Existence results for quasistatic contact problems with Coulomb friction. Appl. Math. Optim. 42 (2000), 169-202. MR1784173 (2001m:74049)

[4] F. Brezzi, M. Fortin, Mixed and Hybrid Finite Element Methods, Vol. 15, Springer Series in Computational Mathematics, Springer-Verlag, 1991. MR.1115205 (92d:65187)

[5] F.H. Clarke, Optimization and Nonsmooth Analysis, Wiley-Interscience Publications, 1983. MR 0709590 (85m:49002)

[6] C. A. de Coulomb, Théorie des machines simples, en ayant égard au frottement de leurs parties et à la roideur des cordages. Pièce qui a remporté le Prix de l'Académie des Sciences pour l'année 1781, Mémoire des Savants Etrangers, X, 1785, pp. 163-332. Reprinted by Bachelier, Paris 1821.

[7] G. Duvaut, Problèmes unilatéraux en mécanique des milieux continus, Actes du Congrès International des Mathématiciens (Nice 1970), Tome 3, Gauthier-Villars, Paris, 1971, pp. 71-77. MR 0426581 (54:14522)

[8] G. Duvaut, J.L. Lions, Les inéquations en mécanique et en physique, Dunod, Paris, 1972. MR 0464857 (57:4778)

[9] C. Eck, J. Jarušek, Existence results for the static contact problem with Coulomb friction. Math. Models Methods Appl. Sci. 8 (1998), 445-468. MR1624879 (99c:73071)

[10] J. Haslinger, Approximation of the Signorini problem with friction, obeying the Coulomb law. Math. Meth. Appl. Sci. 5 (1983), 422-437. MR0716664 (86c:73029)

[11] W. Han, M. Sofonea, Quasistatic Contact Problems in Viscoelasticity and Viscoplasticity, American Mathematical Society, International Press, 2002. MR.1935666 (2003m:74086)

[12] R. Hassani, P. Hild, I. Ionescu, N.-D. Sakki, A mixed finite element method and solution multiplicity for Coulomb frictional contact. Comput. Methods Appl. Mech. Engrg. 192 (2003), 4517-4531. MR2012480 (2004h:74061)

[13] P. Hild, On finite element uniqueness studies for Coulomb's frictional contact model. Appl. Math. Comp. 12 (2002), 41-50. MR1905992 (2003h:74055)

[14] P. Hild, An example of nonuniqueness for the continuous static unilateral contact model with Coulomb friction. C. R. Acad. Sci. Paris 337 (2003), 685-688. MR2030112 (2004i:74085)

[15] P. Hild, Non-unique slipping in the Coulomb friction model in two-dimensional linear elasticity. Q. Jl. Mech. Appl. Math. 57 (2004), 225-235. MR2068404 (2005a:74071)

[16] J.B. Hiriart-Urruty, Tangent cones, generalized gradients and mathematical programming in Banach spaces. Math. of Operations Research 4 (1979), 79-87. MR0543611(80i:58011)

[17] J.B. Hiriart-Urruty, C. Lemaréchal, Convex Analysis and Minimisation Algorithms, Vol. 305, in Grundlehren der Mathematischen Wissenschaften, Springer-Verlag, 1993.

[18] I.R. Ionescu, J.-C. Paumier, On the contact problem with slip rate dependent friction in elastodynamics. Eur. J. Mech., A/Solids 4 (1994), 555-568. MR1298058 (95h:73063)

[19] J. Jarušek, Contact problems with bounded friction. Coercive case. Czechoslovak Math. J. 33 (1983), 237-261. MR0699024 (84h:73045)

[20] H. Khenous, J. Pommier, Y. Renard, Hybrid discretization of the Signorini problem with Coulomb friction. Theoretical aspects and comparison of numerical solvers. To appear in Appl. Numer. Math.

[21] N. Kikuchi, J.T. Oden, Contact problems in elasticity, SIAM, Philadelphia, 1988. MR0961258 (89j:73097)

[22] P. Laborde, Y. Renard, Fixed points strategies for elastostatic frictional contact problems. Submitted, 2003.

[23] J.-L. Lions, E. Magenes, Problèmes aux limites non homogènes. Dunod, Paris, 1968.

[24] J. Nečas, J. Jarušek, J. Haslinger, On the solution of variational inequality to the Signorini problem with small friction. Bolletino U.M.I. 5 (17-B) (1980), 796-811. MR0580559 (82a:49015)

[25] J.-C. Paumier, Bifurcations et méthodes numériques, Masson, Paris, 1997. MR.1474966 (99k:00003)

[26] Y. Renard, Singular perturbation approach to an elastic dry friction problem with a non-monotone friction coefficient. Quart. Appl. Math. 58 (2000), 303-324. MR.1753401 (2001a:74071) 